
\title{
25 Research Soure \\ High-resolution imaging of mitochondria and mitochondrial nucleoids in differentiated SH-SY5Y cells
}

\section{Emily Annuario}

King's College London https://orcid.org/0000-0002-0342-1113

Kristal Y-W Ng

King's College London https://orcid.org/0000-0003-2301-8206

Alessio Vagnoni ( $\square$ alessio.vagnoni@kcl.ac.uk)

King's College London https://orcid.org/0000-0003-2947-9193

\section{Method Article}

Keywords: SH-SY5Y cells, mitochondria, nucleoids, mitochondrial DNA, axonal transport, mitochondrial fission, neuronal differentiation, instant structured illumination microscopy (iSIM), super-resolution

Posted Date: May 24th, 2021

DOI: https://doi.org/10.21203/rs.3.rs-540708/v1

License: (9) This work is licensed under a Creative Commons Attribution 4.0 International License. Read Full License 


\section{Abstract}

Mitochondria are highly dynamic organelles which form intricate networks with complex dynamics. Mitochondrial transport and distribution are essential to ensure proper cell function, especially in cells with an extremely polarised morphology such as neurons. A layer of complexity is added when considering mitochondria have their own genome, packaged into nucleoids. Major mitochondrial morphological transitions, for example mitochondrial division, often occur in conjunction with mitochondrial DNA ( $\mathrm{mtDNA}$ ) replication and changes in the dynamic behaviour of the nucleoids. However, the relationship between mtDNA dynamics and mitochondrial motility in the processes of neurons has been largely overlooked. In this chapter, we describe a method for live imaging of mitochondria and nucleoids in differentiated $\mathrm{SH}-\mathrm{SY} 5 \mathrm{Y}$ cells by instant structured illumination microscopy (iSIM). We also include a detailed protocol for the differentiation of SH-SY5Y cells into cells with a pronounced neuronal-like morphology and show examples of coordinated mitochondrial and nucleoid motility in the long processes of these cells.

Emily Annuario and Kristal Y-W Ng contributed equally to this work.

\section{Introduction}

Neurons are highly polarised cells with long axons and dendrites. Due to their peculiar architecture, neurons are particularly reliant on long-range intracellular transport mechanisms to efficiently distribute organelles, proteins and other cargoes with spatiotemporal precision [1, 2]. This process is crucial to maintain neuronal function, and transport defects have been shown to be involved in several neurodegenerative disorders [3].

Mitochondria are essential neuronal cargoes, whose efficient transport is crucial for maintaining neuronal homeostasis through calcium buffering, metabolite synthesis and provision of ATP at sites with high energy demand, such as synapses [4,5]. Not surprisingly, disruption of mitochondrial transport has been shown to alter neuronal functionality and prime neurons for degeneration [6]. Mitochondria are unique axonal transport cargoes as the majority of them can remain stationary for significant amounts of time, which is unusual for most axonal cargoes [7]. Despite the fact that only a fraction of mitochondria are motile in neurons, the importance of their transport is underscored by the increasing evidence that reduced axonal motility of these organelles can be considered a hallmark of ageing and neurodegeneration [8].

Mitochondria are the only organelles harbouring their own genome, a trace of their prokaryotic origin and symbiotic incorporation into eukaryotic cells $[9,10]$. The mitochondrial genome is distinct from that stored in the nucleus of the cell and encodes 13 essential subunits of respiratory chain complexes, 22 tRNAs and 2 rRNAs necessary for their translation $[11,12]$. The mitochondrial DNA (mtDNA) is located in the mitochondrial matrix and is condensed in small 100-nm [13] nucleoprotein complexes called 
nucleoids, by the mtDNA binding protein mitochondrial transcription factor A (TFAM), a key regulator of mtDNA replication and transcription $[14,15]$.

Live-imaging experiments have shown nucleoids to be highly dynamic structures that are able to redistribute through the mitochondrial network via mitochondrial fission and fusion $[11,16,17]$. Repositioning and/or redistribution of the nucleoids during mitochondrial morphological transitions are intimately linked to the replication of mtDNA. Thus, mtDNA replication marks the site of ER-mediated mitochondrial constriction and subsequent DRP1-dependent fission, thus priming the organelles for division $[18,19]$. On the other hand, mitochondrial fusion aids in the redistribution of mtDNA throughout the mitochondrial network, ensuring mitochondrial integrity and functionality are retained throughout the network [20].

To date, many models for the study of mitochondrial dynamics in neurons have been established in cultured cells and in model organisms. Model organisms are extremely beneficial for in vivo studies of axonal transport in a physiological context [21-31], with the potential added advantage of observing changes that occur during organismal ageing [32-36]. On the other hand, in vitro models for trafficking studies, such as primary neuronal cultures and iPSCs, can be more easily manipulated and afford greater flexibility for pharmacological and genetic manipulation [21, 37-47]. These models, however, have not yet been used extensively to study nucleoid dynamics, except in some instances [48]. Axonal trafficking of nucleoids has rarely been investigated and how mitochondrial dynamics affects the distribution of nucleoids in neuronal cells of human origin is currently unknown.

In this chapter, we describe a protocol to image mitochondrial and nucleoid dynamics in differentiated SH-SY5Y cells. This cell line is a clone of the SK-N-SH neuroblastoma cell line originally isolated from a human metastatic bone marrow [49] and can be differentiated to produce cells that express neuronal markers and have mature neuronal morphology $[50,51]$. The simplicity and efficiency of the differentiation protocol, combined with the human origin of this cell line, the ease of maintenance and mass expansion at relative low cost, make SH-SY5Y cells an advantageous alternative to iPSCs and primary neuronal cultures for routine studies of intracellular trafficking. This system can be easily established in most laboratories and the findings obtained with this system could then be validated in primary neuronal cultures or in model organisms.

\section{Materials}

\subsection{Culture and differentiation of SH-SY5Y cells}

1. SH-SY5Y neuroblastoma cell line (ATCC CRL-2266) routinely cultured in T75 flasks and plated on Ibidi $\mu$-Slide 8 Well Glass Bottom slides (Ibidi, \#80827) coated with $5 \mu \mathrm{g} / \mathrm{cm}^{2}$ Poly-D-Lysine (PDL) for differentiation

2. SH-SY $5 Y$ standard culture medium: add $45 \mathrm{~mL}$ of Heat Inactivated FBS $(10 \% \mathrm{v} / \mathrm{v}$, ThermoFisher \#10500064) and $5 \mathrm{~mL}$ of $200 \mathrm{mM} \mathrm{L-Glutamine,} \mathrm{to} 450 \mathrm{~mL}$ of DMEM (ThermoFisher, supplemented 
with $4.5 \mathrm{~g} / \mathrm{L}$ D-Glucose and $0.11 \mathrm{~g} / \mathrm{L}$ pyruvate)

3. $25 \%$ Trypsin-EDTA

4. Phosphate buffered saline (PBS)

5. Hemocytometer for counting cells

6. Phase I medium: to $47 \mathrm{~mL}$ of DMEM F12 (ThermoFisher, supplemented with $542 \mathrm{mg} / \mathrm{L}$ glutaMAX) add $500 \mu \mathrm{L}$ Penicillin-Streptomycin (10,000 U/mL, 100x), $2.5 \mathrm{~mL}$ of FBS (5\% v/v) and $50 \mu \mathrm{L}$ of EC23 at a final concentration of $10 \mu \mathrm{M}$

7. Phase II medium: to $48.5 \mathrm{~mL}$ of Neurobasal-A (ThermoFisher) add $500 \mu \mathrm{L}$ of $200 \mathrm{mM}$ L-Glutamine, $500 \mu \mathrm{L}$ of N2 Supplement (100x, ThermoFisher), $500 \mu \mathrm{L}$ Penicillin-Streptomycin $(10,000 \mathrm{U} / \mathrm{mL}, 100 \mathrm{x})$ and $2.5 \mu \mathrm{L}$ of BDNF at a final concentration of $50 \mathrm{ng} / \mathrm{mL}$

\subsection{Transfection of SH-SY5Y cells}

1. NeuroMag transfection reagent (OzBiosciences, \#NM50500)

2. Magnetic plate

\subsection{Imaging of cell morphology, mitochondrial and nucleoid transport in differentiated SH-SY5Y cells}

1. Confocal microscope. We use the following settings:

1. For recording cell morphology during differentiation using phase contrast imaging.

Nikon A1R equipped with a PMT detector, a 40x Plan Fluor ELWD Ph2 ADM dry objective (0.6NA) using a Ph2 condenser. Images were acquired upon sample excitation at 513nm (see Note 1). For immunofluorescence images, the same microscope is used with $60 \times \mathrm{CFI}$ Apo oil-immersion objective (1.4 NA)

2. For high-resolution imaging of nucleoid and mitochondrial motility.

Nikon inverted Ti-E equipped with Vt-iSIM head (Vistech), Hamamatsu Orca Flash 4.0sCMOS camera fitted with a 100x CFI TIRF oil-immersion objective (1.49 NA).

For both (a) and (b), imaging was performed at constant temperature of $37^{\circ} \mathrm{C}$ and $5 \% \mathrm{CO}_{2}$ using a microscope enclosure with temperature and $\mathrm{CO}_{2}$ control (Okolab).

2. $1 \mathrm{mM}$ Mitotracker Deep Red (ThermoFisher): add $91.98 \mu \mathrm{L}$ of DMSO to the $50 \mu \mathrm{g}$ of lyophilized Mitotracker. Divide into aliquots and store at $-20^{\circ} \mathrm{C}$.

3. 1:100 dilution of SYBR Gold: $1 \mu \mathrm{L}$ of the commercial stock of SYBR Gold (10'000x, ThermoFisher, \#S11494) is diluted in $99 \mu \mathrm{L}$ DMSO to create the working stock.

4. $1 \mathrm{X}$ CellMask Green staining solution: $1 \mu \mathrm{L}$ of the commercial stock of CellMask Green ( $1{ }^{\prime} 000 \mathrm{X}$, ThermoFisher, \#C37608) is diluted 1:1'000 in culture media to be used to perform CellMask staining.

5. Imaging chambers. 


\subsection{Analysis of mitochondria and nucleoids transport}

1. Fiji/lmage J (https://fiji.sc/ or http://rsbweb.nih.gov/ij/).

2. ImageJ software featuring the following plugins: Stack Reg (Philippe Thevenaz, EPFL, Switzerland), Straighten (Eva Kocsis, NIH, USA) and Velocity Measurement Tool (http://dev.mri.cnrs.fr/projects/imagej-macros/wiki/Velocity_Measurement_Tool).

\section{Methods}

The SH-SY5Y cell line was shown to be composed of two cellular subpopulations: substrate adherent epithelial-like (S-type) and neuroblastic ( $\mathrm{N}$-type) cells, with the latter undergoing differentiation to produce neuronal-like cells [50,52]. Currently, a number of differentiation protocols exist which utilise various neuralising agents that can drive differentiation toward a more cholinergic, adrenergic, or dopaminergic phenotypes $[53,54]$. Due to the versatility of the differentiation protocols, SH-SY5Y cells have been extensively used as disease models for different neurological disorders. They have been largely employed to model several aspects of Parkinson's disease biology $[53,55,56]$, but they were also exploited to study Alzheimer's disease-related insults [57] and neurodevelopment in the context of psychiatric disorders [58].

Retinoic Acid (RA)-mediated differentiation protocols have been shown to induce differentiation into cells with higher choline acetyltransferase activity [59-61], while other studies have focused on a more dopaminergic phenotype [62]. RA has also been often used in combination with other agents, such as 120-tetradecanoylphorbol-13-acetate (TPA), for a more efficient induction of cells with a dopaminergic phenotype, for example increased expression of tyrosine hydroxylase (TH), the dopamine transporter (DAT), and dopamine receptor subtypes 2 and 3 [63-65]. If desirable, treating SH-SY5Y cells with siRNA targeting the splicing factor PTBP1 prior to differentiation could be used to potentiate neurite outgrowth, as reported for SH-SY5Y cybrids [66].

For the purpose of studying trafficking, the combination of RA treatment with decreasing amounts of serum, followed by the addition of neurotrophic factors such as BDNF, has been shown to be particularly effective in promoting neurite extension during neuronal differentiation [67-69], with the end point of differentiation defined as commitment to a neuronal phenotype with long extended processes [70]. SHSY5Y cells expressing a truncated form of spastin have been used to study the trafficking of synaptophysin-positive vesicles [71]. Furthermore, mitochondrial trafficking was found to be perturbed in SH-SY5Y cells differentiated with RA and BDNF for 8 days in vitro and overexpressing the A53T asynuclein mutant [68], indicating the suitability of the differentiation protocol to study trafficking in neuronal processes.

We differentiated SH-SY5Y cells by adapting a protocol published by Forster et al [69], which requires the addition of neuralising agents such as EC23 (a synthetic analog of all-trans retinoic acid [72-74]) and BDNF to sustain neurite outgrowth (Figure 1A). Coating matrices, seeding densities and time in vitro were optimised to ensure the morphology of the cells was suitable for neurite tracking, i.e. cultures sparse 
enough to visualise long processes derived from a specific cell body but without compromising their survival. Morphological assessments via phase contrast image acquisition (Figure 1B) and staining of plasma membrane compartments (Figure 1C), immunocytochemistry (ICC) and biochemical analysis (Figure 2) validate our differentiation protocol. Figure 1B shows the progression of typical SH-SY5Y cultures throughout the differentiation protocol, with a noticeable increase in neurite extension, accompanied by clustering of cellular somata, already visible around DIV7 and with a clear network of neuronal-like processes around DIV14 (see Note 2). Figure 2 show substantial upregulation of neuronal markers (neurofilaments, MAP2, Figure 2A-C), including markers for catecholaminergic neurons (tyrosine hydroxylase, Figure 2D) in differentiated cells compared to undifferentiated SH-SY5Y. A significant increase in the number of axonal-like, neurofilament positive neurites can be observed, which were also significantly longer than the sparser processes extended by undifferentiated cells (Figure 2B-C).

SH-SY5Y cells stained with the SYBR Gold nucleotide dye at low concentration [75] display a discrete punctuated pattern that colocalises with the mitochondrial network (Figure 3A), indicative of specific nucleoid staining. Overexpression of the fluorescently tagged nucleoid protein TFAM, a major component of mammalian nucleoids and routinely used as nucleoid marker, results in extensive co-localisation between TFAM and the SYBR signals (Figure 3B), thus confirming SYBR Gold as a suitable dye for the visualisation of nucleoids in these cells. In the neurites of differentiated cells, mitochondria and nucleoids exhibit robust motility with most mitochondria containing at least one nucleoid when observed by live-cell iSIM imaging (Figures 4 and 5 and Movies 1 and 2). Differentiated SH-SY5Y cells have been recently used for imaging the movement of synaptic vesicles by single-molecule localization microscopy [76]. Our protocol shows the suitability of this system for studying long-range co-transport of mitochondria and nucleoids by live super-resolution imaging. The method also lends itself to studying the partitioning and distribution of nucleoids during mitochondrial dynamics in the neurites of the cells. Figures 4 and 5 show that nucleoids can be distributed differently into daughter mitochondria during fission in the processes, and mitochondria devoid of nucleoids can arise after mitochondrial division, as recently shown in Cos-7 cells and mouse cardiomyocytes [77].

\subsection{Differentiation of SH-SY5Y cells into neuronal-like cells}

1. On the first day of the differentiation protocol, coat the wells of an 8 well Ibidi chamber with PDL matrix by adding $4 \mu \mathrm{L}$ of $10 \mathrm{mg} / \mathrm{mL}$ PDL to $2 \mathrm{~mL}$ autoclaved MilliQ water and incubate at $37^{\circ} \mathrm{C}$ for at least 2 hours. We coat each Ibidi well $\left(1 \mathrm{~cm}^{2}\right)$ with $250 \mu \mathrm{L}$ of matrix solution (see Note 3 ).

2. Wash the wells $3 x$ times with PBS and allow them to dry out prior to seeding cells. Ensuring the PDL has dried out will improve cell adhesion.

3. Add $1 \mathrm{~mL}$ of Trypsin-EDTA (approximately $0.014 \mathrm{ml} / \mathrm{cm}^{2}$ ) to a T75 flask of undifferentiated SH-SY5Y cells and leave for 3 minutes to detach the cells.

4. Add $4 \mathrm{~mL}$ of standard media to detached cells (total $5 \mathrm{~mL}$ ), collect the cell suspension in a falcon tube, spin at $300 \mathrm{~g}$ for 3 minutes and resuspend the pellet in $3 \mathrm{~mL}$. 
5. Make a 1:5 dilution of cells in Trypan blue (50 $\mu \mathrm{L}$ cells plus $200 \mu \mathrm{L}$ Tyrpan Blue) and count cells with an hemocytometer.

6. Seed SH-SY5Y cells at a density of $15^{\prime} 000 \mathrm{cells} / \mathrm{cm}^{2}$ and keep them in their standard culture medium overnight (300 $\mu \mathrm{L} /$ well) (see Note 4$)$.

7. The following day change the standard medium to $300 \mu \mathrm{L}$ of Phase I medium containing the retinoic acid receptor agonist EC23. This starts the differentiation process and is referred to as DIV 1 (Figure $1 \mathrm{~A})$.

8. After 72 hours, on DIV 4, change medium to $300 \mu \mathrm{L}$ Phase II containing BDNF to sustain neurite outgrowth. This is the medium used throughout the rest of the experiment and is changed every 3 to 4 days (see Note 5).

9. Assess cell differentiation daily by brightfield or phase contrast microscopy (Fig. 1B). At key timepoints (Figure 1A), markers of cell differentiation can be verified by ICC, western blotting and CellMask staining (Fig. 2 and see Note 6).

\subsection{Transfection of SH-SY5Y cells}

If transfection is required to visualise fluorescent organelles or proteins, we suggest performing it 48 hours before imaging (for instance, DIV 12 for imaging DIV 14). For each well of an Ibidi $\mu$-Slide 8-well Glass Bottom slide:

1. Seed the cells as described in section 3.1 for the transfection of differentiated cells. For transfection of undifferentiated cells, cells are plated the day before at a density of $55^{\prime} 000 / \mathrm{cm}^{2}$.

2. Tube 1: Mix 0.6 $\mu \mathrm{L}$ of NeuroMag in $24.4 \mu \mathrm{L} 1 \mathrm{x}$ PBS.

3. Tube 2: Mix $0.3 \mu \mathrm{g}$ of DNA in a final volume of $25 \mu \mathrm{L} 1 \times$ PBS and flick tube to mix DNA.

4. Add the contents of the tubes together, mix well by flicking and leave to incubate for 20 minutes in the hood. The ratio of NeuroMag to DNA is 2:1 in a volume of $50 \mu \mathrm{L}$ (see Note 7). This ratio should be maintained if scaling experiments into different vessels.

5. Remove enough conditioned media from cells to leave a volume of $200 \mu \mathrm{L}$ in each well.

6. Add the transfection solution dropwise onto cells, ensuring it spreads evenly within the well and incubate at $37^{\circ} \mathrm{C}$ for 30 minutes on the magnetic plate.

7. Remove the magnetic plate and leave the transfection mix to incubate for 48 hours, until imaging or subsequent experiments.

\subsection{Imaging transport of mitochondria and nucleoids in differentiated SH-SY5Y cells}

1. One hour before imaging, prepare the medium containing dyes by thawing the aliquots and diluting as follows (we recommend preparing serial dilutions):

1. 1:5'000 of the $1 \mathrm{mM}$ Mitotracker Deep Red, for a final solution of $200 \mathrm{nM}$ in imaging media

2. 1:2'000 of the previously made 1:100 SYBR Gold stock 
2. Remove media from wells and gently add $300 \mu \mathrm{L} /$ well of media containing dyes per well. Leave the cells with dyes in the incubator for 30 minutes.

3. During incubation, set the microscope temperature to $37^{\circ} \mathrm{C}$ and $\mathrm{CO}_{2}$ to $5 \%$ (see Note 8).

4. After incubation, remove the dyes and wash wells $3 x$ times with PBS to remove excess dye, which might otherwise aspecifically stain other non-mitochondrial compartments (see Note 9).

5. Once finished with the washes, add fresh Phase II media to the cells.

6. Go to the microscope and image the cells.

7. Focus on the cells by using the transmitted light (through eyepiece) and scan the well to find an appropriate area which contains a good number of processes that can be traced back to their cell bodies (see Note 10).

8. Switch to confocal mode (through microscope software) and observe the MitoTracker Deep Red signal on the computer via the $640 \mathrm{~nm}$ laser. Fine tune the focus and laser settings so that the mitochondria are clearly visible in the region of interest. Do the same for the SYBR Gold signal with the $488 \mathrm{~nm}$ laser (see Note 11 and Note 12).

9. Image the region of interest at a rate of $1 \mathrm{fps}$ for 1 minute (see Note 13). This will be saved as a ".nd2" file that contains two channels and 61 timeframes.

\subsection{Analysis of mitochondria and nucleoids transport}

1. Open the ".nd2" file with Fiji/ImageJ. Adjust the intensity settings with the "Brightness/Contrast" tool, if needed (Image> Adjust> Brightness/Contrast).

2. Inspect the images for any potential sample drifts that may have occurred during imaging. Align image frames using the StackReg plugin to correct the drift, if necessary.

3. Identify process you wish to analyse, select it with the "Segmented" or "Freehand" (right click to change in Line Selection Tool) and use the Straighten plugin to straighten it. When the pop-up window appears, adjust the "Line width" to ensure it includes the whole width of your process and select "Process Entire Stack". This will open a new window which shows the whole movie of the straightened process; the direction from which you select the process will result in its orientation in this window.

4. The straightened segment can now be used to create a kymograph with the Velocity Measurement Tool. Once the plugin has been launched, retrace the straightened process ("Straight line", press Shift while selecting to keep line straight) and press the " $k$ " button which will have appeared on the toolbar (see Note 14).

\section{Notes}

1. Phase contrast imaging was performed on live samples at a constant temperature of $37^{\circ} \mathrm{C}$ and $5 \%$ $\mathrm{CO}_{2}$. We feel the use of live cells rather than fixed sample gives a more accurate representation of the culture as a whole, especially in preparation for the trafficking experiments in live cells. 
2. Formation of clear neuronal-like neurites was observed between DIV 10 and DIV 17, without a discernible difference in neurite length between DIV 14 and DIV 21. With this protocol, we found that cultures at DIV 21 started to appear less healthy, with a reduced number of processes and clusters of somata. They also tended to detach more easily from the glass surface during media changes. Thus, DIV 10-17 was determined to be the best interval for imaging experiments. Within this interval, we recommend using cells at the same DIV throughout an experimental series. We did not observe a decrease in the differentiation efficiency of SH-SY5Y cells cultured for many passages $(>40$ passages in vitro).

3. We also tested coating the wells with $10 \mu \mathrm{g} / \mathrm{mL}$ laminin [78] but no significant differences in cell morphology and neurite extension were noticed when compared to PDL.

4. We use Ibidi $\mu$-Slide 8-well Glass Bottom slides for live imaging experiments. In these chambers, the area of a well is $1 \mathrm{~cm}^{2}$, therefore the seeding density will be $15^{\prime} 000$ cells/well. We tested a range of seeding densities to obtain cultures where the cells are sparse enough for the purpose of mitochondria and nucleoid tracking within single processes and to be able to unequivocally assign a process to the cell body it emanates from. At the same time, the cells need to be seeded at a sufficient density as to not compromise cell survival. In 8-well Ibidi slides, densities lower than 10'000 cells $/ \mathrm{cm}^{2}$ lead to poor growth and occasional cell death after few days of culture, while densities above $20^{\prime} 000$ cells $/ \mathrm{cm}^{2}$ result in cultures that are too compact for clear imaging of single processes. We recommend testing a range of seeding concentrations to find the optimal density for imaging studies.

5. Because retinoic acid is used to reduce cell proliferation and induce neuronal differentiation, we tested leaving the cells in Phase I media for an additional 72 hours (i.e., throughout DIV 1-6) to prolong the incubation in EC23. Only at this point (DIV 7), we changed to Phase II. We expected this alternative protocol to bring forward the point of optimal differentiation. Surprisingly, we found that the culture reached full confluency by DIV 6 with many cells detaching during media changes. As a result, these cultures badly withstood transfection, with the majority of cells detaching prior to imaging.

It is worth noting that two cellular subtypes exist within the SH-SY5Y population: a predominant N-type cell population which becomes non-proliferative upon differentiation, and a small S-type subpopulation that continues to proliferate. Different differentiation methods can result in a different balance of proliferative and non-proliferative cell types [50,52].

6. For ICC analysis, cells were grown on coverslips, fixed in ice-cold methanol for 3 minutes, permeabilized in $0.2 \%$ Triton X-100 for 10 minutes and blocked in $5 \%$ goat serum for 30 minutes. Cells were then stained with anti-neurofilament (1:100, BioLegend, \#837904) and anti-MAP2 (1:250, GeneTex, GTX82661) primary antibodies for 1.5 hours at room temperature. After washing $3 x$ times with PBS, the coverslips were incubated with Alexa Fluor secondary antibodies (1:2'000) for 45 minutes at room temperature, followed by a 5-minute wash in Hoechst $\left(1: 5^{\prime} 000\right)$ and three more PBS washes. Coverslips were mounted with Prolong Gold Antifade mounting solution. Neurite analysis 
was performed in Fiji using the NeuronJ plugin

(ttp://imagescience.org/meijering/software/neuronj/).

For western blotting analysis, $5 \mu \mathrm{g}$ of protein were run on $4-12 \%$ Bis-Tris acrylamide gels and transferred on PVDF membranes for 1-1.5 hours at $35 \mathrm{~V}$. The membranes were incubated with anti-GAPDH (1:5'000, ThermoFisher) and anti-TH (1:200, Abcam) primary antibodies overnight at $4^{\circ} \mathrm{C}$. Membranes were then incubated with HRP-conjugated secondary antibodies (1:5'000, Amerhsam) for 1.5 hours at room temperature, before being imaged with a Bio-Rad Chemidoc Imager. Exposure times to detect specific immunoreactive protein bands were set between 5 and 10 minutes.

For plasma membrane staining with CellMask, cells were incubated with $300 \mu \mathrm{L} /$ well of culture media supplemented with a 1:1'000 dilution of the commercial CellMask Green stock for 30 minutes. Prior to imaging, the dye was removed, wells were washed $3 x$ times with PBS to remove excess dye, and kept in Phase II media post-wash and during imaging.

7. PEI and Lipofectamine were also tested during the optimisation of the transfection protocol but using NeuroMag resulted in higher transfection rate and healthier cell cultures. The transfection rate is significantly reduced in differentiated cells with all reagents, which is a further confirmation of the neuronal identity of these cells. We recommend using lentiviral transduction of differentiated cells for high expression of exogenous genes.

8. It is advisable to turn on the temperature and $\mathrm{CO}_{2}$ control with enough time before the start of the imaging session to allow the system to reach the desired temperature $\left(37^{\circ} \mathrm{C}\right)$ and $\mathrm{CO}_{2}(5 \%)$.

9. Be gentle while washing off the excess dye with PBS by pipetting into the side of the well as to not mechanically detach the cells from the bottom of the well, especially at higher DIV. From DIV 16-17, the cells appear to come off more easily during media changes.

10. Finding a field of view where neurites can be traced back to their cell body with ease is essential for analysing anterograde and retrograde trafficking (i.e., away from and towards the cell soma, respectively). Using a cell membrane dye such as CellMask (Figure 1C) could aid in doing so (see

\section{Note 6).}

11. In time-lapse iSIM experiments in HeLa cells, SYBR Gold was used at a 1:10'000 dilution of the commercial stock (i.e., 1x) [75]. In our setup, we found that the range of SYBR dilution that yielded specific and visible puncta varied between 1:100'000 and 1:1'000'000 of the commercial stock (i.e., $0.1 \mathrm{x}-0.01 \mathrm{x}$ ). With further dilutions, the SYBR stain was barely detectable, while higher concentrations often produced bright and nonspecific aggregates. We recommend optimisation of the working dilution prior to live imaging experiments.

12. SYBR Green [17] and PicoGreen [79] have been previously used as mtDNA dye. We therefore tested PicoGreen (Invitrogen, P7581) in addition to SYBR Gold. We observed co-localisation between overexpressed TFAM and PicoGreen-positive puncta at concentrations as low as $1.5 \mu \mathrm{L} / \mathrm{mL}$, which confirms the suitability of this dye to stain nucleoids in SH-SY5Y cells. SYBR Gold was preferred for quantification of trafficking and dynamics due to the higher resolution and more defined puncta 
observed. As with SYRB Gold, the DIV of the cells affects the quality of PicoGreen staining, with cultures at higher DIV more commonly showing non-specific staining in the form of larger extracellular accumulations of dye.

13. To avoid bleaching, find the lower exposure time and laser power at which the mitochondria and the nucleoids are clearly detectable. We observe minimal phototoxicity with our settings of $30 \%$ laser power, 100 ms exposure time for both channels and $1 \mathrm{fps}$ acquisition rate. A high acquisition rate captures more detailed movements which is beneficial when observing the dynamics of small structures such as nucleoids, although it comes at the expense of phototoxicity.

14. Other plugins and kymograph analysis bundles are suitable to produce Kymographs, for instance, KymographBuilder (http://imagej.net/KymoResliceWide), KymoToolBox (https://github.com/fabricecordelieres/IJ-Plugin_KymoToolBox), KymoClear and KymoDirect [80], KymoAnalyzer [81] or KymoButler [82].

\section{Declarations}

\section{Acknowledgements}

This work was funded through a NC3Rs David Sainsbury Fellowship and SKT grants (NC/N001753/2 and NC/T001224/1), a van Geest Fellowship in Dementia and Neurodegeneration from King's College London, a Royal Society Research Grant (RGS R2 180450 ), an Academy of Medical Sciences Springboard Award (SBF004\1088) and a BBSRC CTP Studentship (BB/T508597/1) to AV.

We would like to thank the Wohl Cellular Imaging Centre at King's College London for help with light microscopy and Ricky Patel (King's College London) for sharing an early adapted version of the differentiation protocol from Forster et al [69].

This manuscript will be included in the forthcoming volume of Methods in Molecular Biology on 'Axonal transport'.

\section{Competing interests}

The authors declare no competing interests.

\section{References}

1.Guedes-Dias P, Holzbaur ELF (2019) Axonal transport: Driving synaptic function. Science 366:eaaw9997. https://doi.org/10.1126/science.aaw9997

2 Surana S, Villarroel-Campos D, Lazo OM, et al (2020) The evolution of the axonal transport toolkit. Traffic 21:13-33. https://doi.org /10.1111/tra.12710 
3.Sleigh JN, Rossor AM, Fellows AD, et al (2019) Axonal transport and neurological disease. Nat. Rev. Neurol. 15:691-703. https://doi.org/10.1038/s41582-019-0257-2

4.Chamberlain KA, Sheng ZH (2019) Mechanisms for the maintenance and regulation of axonal energy supply. J. Neurosci. Res. 97:897-913. https://doi.org/10.1002/jnr.24411

5.Misgeld T, Schwarz TL (2017) Mitostasis in Neurons: Maintaining Mitochondria in an Extended Cellular Architecture. Neuron 96:651-666. https://doi.org/10.1016/j.neuron.2017.09.055

6.Sheng Z-H, Cai Q (2012) Mitochondrial transport in neurons: impact on synaptic homeostasis and neurodegeneration. Nat Rev Neurosci 13:77-93. https://doi.org/10.1038/nrn3156

7.Hollenbeck PJ, Saxton WM (2005) The axonal transport of mitochondria. J Cell Sci 118:5411-5419. https://doi.org/10.1242/jcs.02745

8.Mattedi F, Vagnoni A (2019) Temporal Control of Axonal Transport: The Extreme Case of Organismal Ageing. Front Cell Neurosci 13:393. https://doi.org/10.3389/fncel.2019.00393

9.Sagan L (1967) On the origin of mitosing cells. J Theor Biol 14: 255-274. https://doi.org/10.1016/00225193(67)90079-3

10.Gray MW (2017) Lynn Margulis and the endosymbiont hypothesis: 50 years later. Mol Biol Cell 28:1285-1287. https://doi.org/10.1091/mbc.E16-07-0509

11.Nicholls TJ, Gustafsson CM (2018) Separating and Segregating the Human Mitochondrial Genome. Trends Biochem. Sci. 43:869-881. https://doi.org/10.1016/j.tibs.2018.08.007

12.Taanman JW (1999) The mitochondrial genome: Structure, transcription, translation and replication. Biochim. Biophys. Acta - Bioenerg. 1410:103-123. https://doi.org/10.1016/S0005-2728(98)00161-3

13.Kukat C, Wurm CA, Spåhr H, et al (2011) Super-resolution microscopy reveals that mammalian mitochondrial nucleoids have a uniform size and frequently contain a single copy of mtDNA. Proc Natl Acad Sci U S A 108:13534-13539. https://doi.org/10.1073/pnas.1109263108

14.Farge GÉR, Falkenberg M (2019) Organization of DNA in mammalian mitochondria. Int. J. Mol. Sci. 20: 2770 . https://doi.org/10.3390/ijms20112770

15.Chapman J, Ng YS, Nicholls TJ (2020) The maintenance of mitochondrial DNA integrity and dynamics by mitochondrial membranes. Life 10:1-42. https://doi.org/10.3390/life10090164

16.Chen Z, Wang ZH, Zhang G, et al (2020) Mitochondrial DNA segregation and replication restrict the transmission of detrimental mutation. J Cell Biol 219: e201905160.

https://doi.org/10.1083/JCB.201905160 
17.Ban-Ishihara R, Ishihara T, Sasaki N, et al (2013) Dynamics of nucleoid structure regulated by mitochondrial fission contributes to cristae reformation and release of cytochrome c. Proc Natl Acad Sci U S A 110:11863-11868. https://doi.org/10.1073/pnas.1301951110

18.Garrido N, Griparic L, Jokitalo E, et al (2003) Composition and dynamics of human mitochondrial nucleoids. Mol Biol Cell 14:1583-1596. https://doi.org/10.1091/mbc.E02-07-0399

19.Lewis SC, Uchiyama LF, Nunnari J (2016) ER-mitochondria contacts couple mtDNA synthesis with Mitochondrial division in human cells. Science 353:aaf5549. https://doi.org/10.1126/science.aaf5549

20.Ramos ES, Motori E, Brüser C, et al (2019) Mitochondrial fusion is required for regulation of mitochondrial DNA replication. PLoS Genet 15:e1008085. https://doi.org/10.1371/journal.pgen.1008085

21.Turchetto S, Le Bail R, Broix L, Nguyen L (2021) Molecular analysis of axonal transport dynamics upon modulation of microtubule acetylation. Methods Mol Biol This volume.

22.Sleigh JN, Vagnoni A, Twelvetrees AE, Schiavo G (2017) Methodological advances in imaging intravital axonal transport. F1000Research 6:200. https://doi.org/10.12688/f1000research.10433.1

23.Mattedi F, Chennell G, Vagnoni A (2021) Detailed imaging of mitochondrial transport and precise manipulation of mitochondrial function with genetically-encoded photosensitizers in adult Drosophila neurons. Methods Mol Biol This volume.

24. Huang R, Xu Y, Chen M, et al (2021) Visualizing the intracellular trafficking in zebrafish Mauthner cells. Methods Mol Biol This volume.

25.Atkins $M$, Hazan J, Fassier C (2021) In vivo live imaging of axonal transport in developing zebrafish axons. Methods Mol Biol This volume.

26.Barmaver SN, Shanmugam MM, Wagner OI (2021) Methods to quantify and relate axonal transport defects to changes in C. elegans behavior. Methods Mol Biol This volume.

27.Knabbe J, Protzmann J, Kuner T (2021) In vivo imaging of axonal organelle transport in the mouse brain. Methods Mol Biol This volume.

28.Anazawa Y, Niwa S (2021) Analyzing the impact of gene mutations on axonal transport in Caenorhabditis elegans. Methods Mol Biol This volume.

29.Robinson W, Godenschwege TA (2021) Live imaging of axonal transport in the adult drosophila central nervous system. Methods Mol Biol This volume.

30.Nadiminti SSP, Koushika SP (2021) Imaging intracellular trafficking in neurons of C. elegans. Methods Mol Biol This volume. 
31.Bearer E, Zhang X, Jacobs R (2021) Studying Axonal Transport in the Brain by Manganese-Enhanced Magnetic Resonance Imaging (MEMRI). Methods Mol Biol This volume.

32.Vagnoni A, Hoffmann PC, Bullock SL (2016) Reducing Lissencephaly-1 levels augments mitochondrial transport and has a protective effect in adult Drosophila neurons. J Cell Sci 129:178-190.

https://doi.org/10.1242/jcs.179184

33.Vagnoni A, Bullock SL (2018) A cAMP/PKA/Kinesin-1 Axis Promotes the Axonal Transport of Mitochondria in Aging Drosophila Neurons. Curr Biol 28:1265-1272.e4.

https://doi.org/10.1016/j.cub.2018.02.048

34.Morsci NS, Hall DH, Driscoll M, Sheng Z-H (2016) Age-Related Phasic Patterns of Mitochondrial Maintenance in Adult Caenorhabditis elegans Neurons. J Neurosci 36:1373-1385. https://doi.org/10.1523/JNEUROSCI.2799-15.2016

35.Takihara $\mathrm{Y}$, Inatani M, Eto $\mathrm{K}$, et al (2015) In vivo imaging of axonal transport of mitochondria in the diseased and aged mammalian CNS. Proc Natl Acad Sci U S A 112:10515-20.

https://doi.org/10.1073/pnas.1509879112

36.Sleigh JN, Tosolini AP, Gordon D, et al (2020) Mice Carrying ALS Mutant TDP-43, but Not Mutant FUS, Display In Vivo Defects in Axonal Transport of Signaling Endosomes. Cell Rep 30:3655-3662.e2. https://doi.org/10.1016/j.celrep.2020.02.078

37.Tierney W, Vicino I, Sun S, et al (2021) Methods and Applications of Campenot Trichamber Neuronal Cultures for the Study of Neuroinvasive Viruses. Methods Mol Biol This volume.

38. Voelzmann A, Sanchez-Soriano N (2021) Drosophila primary neuronal cultures as a useful cellular model to study and image axonal transport. Methods Mol Biol This volume.

39.Annuario EA, Ng KY-W (2021) High-resolution live imaging of mitochondrial nucleoids in differentiated SH-SY5Y cells. Methods Mol Biol This volume.

40.Ganguly A, Roy S (2021) Imaging diversity in slow axonal transport. Methods Mol Biol This volum:

41.Badal K, Zhao Y, Miller K, Puthanveettil S (2021) Live imaging and quantitative analysis of organelle transport in sensory neurons of Aplysia Californica. Methods Mol Biol This volume.

42. Hees TJ, Harbauer AB (2021) Live-cell imaging of RNA transport in axons of cultured primary neurons. Methods Mol Biol This volume.

43.Shekari A, Fahnestock M (2021) Retrograde Axonal Transport of Neurotrophins in Basal Forebrain Cholinergic Neurons. Methods Mol Biol This volume. 
44.Ionescu A, Perlson E (2021) Microfluidic neuromuscular co-culture system for tracking cell-to-cell transfer and axonal transport of labeled proteins. Methods Mol Biol This volume.

45.Montgomery A, Garbouchian A, Bentley M (2021) Visualizing vesicle-bound kinesins in cultured hippocampal neurons. Methods Mol Biol This volume.

46.Emily MF, Agrawal L, Barzaghi P, et al (2021) Use of microfluidics chambers to image axonal transport in adult sensory neurons. Methods Mol Biol This volume.

47.Mehta AR, Chandran S, Selvaraj BT (2021) Assessment of mitochondrial trafficking as a surrogate of fast axonal transport in human induced pluripotent stem cell derived spinal motor neurons. Methods Mol Biol This volume.

48.Bateman JM (2021) Mitochondrial DNA transport in Drosophila neurons. Methods Mol Biol This volume.

49.Biedler JL, Helson L, Spengler BA (1973) Morphology and Growth, Tumorigenicity, and Cytogenetics of Human Neuroblastoma Cells in Continuous Culture. Cancer Res 33:2643-52.

50.Encinas M, Iglesias M, Liu Y, et al (2000) Sequential treatment of SH-SY5Y cells with retinoic acid and brain-derived neurotrophic factor gives rise to fully differentiated, neurotrophic factor-dependent, human neuron-like cells. J Neurochem 75:991-1003. https://doi.org/10.1046/j.1471-4159.2000.0750991.x

51.Shipley MM, Mangold CA, Szpara ML (2016) Differentiation of the SH-SY5Y human neuroblastoma cell line. J Vis Exp (108):53193. https://doi.org/10.3791/53193

52.Ross RA, Spengler BA, Biedler JL (1983) Coordinate Morphological and Biochemical Interconversion of Human Neuroblastoma Cells. J Natl Cancer Inst 71:741-747. https://doi.org/10.1093/jnci/71.4.741

53.Xicoy H, Wieringa B, Martens GJM (2017) The SH-SY5Y cell line in Parkinson's disease research: a systematic review. Mol. Neurodegener. 12:1-11

54.Kovalevich J, Langford D (2013) Considerations for the use of SH-SY5Y neuroblastoma cells in neurobiology. Methods Mol Biol 1078:9-21. https://doi.org/10.1007/978-1-62703-640-5_2

55.Xie W, Chung KKK (2012) Alpha-synuclein impairs normal dynamics of mitochondria in cell and animal models of Parkinson's disease. J Neurochem 122:404-414. https://doi.org/10.1111/j.14714159.2012.07769.x

56.Ito K, Eguchi Y, Imagawa Y, et al (2017) MPP+ induces necrostatin-1-and ferrostatin-1-sensitive necrotic death of neuronal SH-SY5Y cells. Cell Death Discov 3:.

https://doi.org/10.1038/cddiscovery.2017.13 
57.de Medeiros LM, De Bastiani MA, Rico EP, et al (2019) Cholinergic Differentiation of Human Neuroblastoma SH-SY5Y Cell Line and Its Potential Use as an In vitro Model for Alzheimer's Disease Studies. Mol Neurobiol 56:7355-7367. https://doi.org/10.1007/s12035-019-1605-3

58.Chiocchetti AG, Haslinger D, Stein JL, et al (2016) Transcriptomic signatures of neuronal differentiation and their association with risk genes for autism spectrum and related neuropsychiatric disorders. Transl Psychiatry 6:. https://doi.org/10.1038/tp.2016.119

59.Påhlman S, Ruusala Al, Abrahamsson L, et al (1984) Retinoic acid-induced differentiation of cultured human neuroblastoma cells: a comparison with phorbolester-induced differentiation. Cell Differ 14:135144. https://doi.org/10.1016/0045-6039(84)90038-1

60.Adem A, Mattsson MEK, Nordberg A, Påhlman S (1987) Muscarinic receptors in human SH-SY5Y neuroblastoma cell line: regulation by phorbol ester and retinoic acid-induced differentiation. Dev Brain Res 33:235-242. https://doi.org/10.1016/0165-3806(87)90156-8

61.Hashemi SH, Li JY, AhIman H, Dahlström A (2003) SSR2(a) receptor expression and adrenergic/cholinergic characteristics in differentiated SH-SY5Y cells. Neurochem Res 28:449-460. https://doi.org/10.1023/A:1022848718109

62.Korecka JA, van Kesteren RE, Blaas E, et al (2013) Phenotypic Characterization of Retinoic Acid Differentiated SH-SY5Y Cells by Transcriptional Profiling. PLoS One 8:.

https://doi.org/10.1371/journal.pone.0063862

63.Presgraves SP, Ahmed T, Borwege S, Joyce JN (2003) Terminally differentiated SH-SY5Y cells provide a model system for studying neuroprotective effects of dopamine agonists. Neurotox Res 5:579-598. https://doi.org/10.1007/BF03033178

64.Constantinescu R, Constantinescu AT, Reichmann H, Janetzky B (2007) Neuronal differentiation and long-term culture of the human neuroblastoma line SH-SY5Y. In: Neuropsychiatric Disorders An Integrative Approach. Springer Vienna, pp 17-28

65.Lopes FM, Schröder R, Júnior MLC da F, et al (2010) Comparison between proliferative and neuron-like SH-SY5Y cells as an in vitro model for Parkinson disease studies. Brain Res 1337:85-94. https://doi.org/10.1016/j.brainres.2010.03.102

66.Pignataro D, Francia S, Zanetta F, et al (2017) A missense MT-ND5 mutation in differentiated Parkinson Disease cytoplasmic hybrid induces ROS-dependent DNA Damage Response amplified by DROSHA. Sci Rep 7:. https://doi.org/10.1038/s41598-017-09910-x

67.Mastroeni D, Grover A, Leonard B, et al (2009) Microglial responses to dopamine in a cell culture model of Parkinson's disease. Neurobiol Aging 30:1805-1817.

https://doi.org/10.1016/j.neurobiolaging.2008.01.001 
68.Melo TQ, van Zomeren KC, Ferrari MFR, et al (2017) Impairment of mitochondria dynamics by human A53T a-synuclein and rescue by NAP (davunetide) in a cell model for Parkinson's disease. Exp Brain Res 235:731-742. https://doi.org/10.1007/s00221-016-4836-9

69.Forster Jl, Köglsberger S, Trefois C, et al (2016) Characterization of differentiated SH-SY5Y as neuronal screening model reveals increased oxidative vulnerability. J Biomol Screen 21:496-509. https://doi.org/10.1177/1087057115625190

70.Shipley MM, Mangold CA, Kuny C V., Szpara ML (2017) Differentiated Human SH-SY5Y Cells Provide a Reductionist Model of Herpes Simplex Virus 1 Neurotropism. J Virol 91:. https://doi.org/10.1128/jvi.00958-17

71.Leo L, Weissmann C, Burns M, et al (2017) Mutant spastin proteins promote deficits in axonal transport through an isoform-specific mechanism involving casein kinase 2 activation. Hum Mol Genet 26:2321-2334. https://doi.org/10.1093/hmg/ddx125

72.Clemens G, Flower KR, Henderson AP, et al (2013) The action of all-trans-retinoic acid (ATRA) and synthetic retinoid analogues (EC19 and EC23) on human pluripotent stem cells differentiation investigated using single cell infrared microspectroscopy. Mol Biosyst 9:677-692. https://doi.org/10.1039/c3mb25505k

73.Christie VB, Barnard JH, Batsanov AS, et al (2008) Synthesis and evaluation of synthetic retinoid derivatives as inducers of stem cell differentiation. Org Biomol Chem 6:3497-3507. https://doi.org/10.1039/b808574a

74.Palomares T, Cordero M, Bruzos-Cidon C, et al (2018) The neuroprotective effect of conditioned medium from human adipose-derived mesenchymal stem Cells is impaired by $\mathrm{N}$-acetyl cysteine supplementation. Mol Neurobiol 55:13-25. https://doi.org/10.1007/s12035-017-0714-0

75.Jevtic V, Kindle P, Avilov S V. (2018) SYBR Gold dye enables preferential labelling of mitochondrial nucleoids and their time-lapse imaging by structured illumination microscopy. PLoS One 13:. https://doi.org/10.1371/journal.pone.0203956

76. Halabi EA, Pinotsi D, Rivera-Fuentes P (2019) Photoregulated fluxional fluorophores for live-cell superresolution microscopy with no apparent photobleaching. Nat Commun 10:. https://doi.org/10.1038/s41467-019-09217-7

77.Kleele T, Rey T, Winter J, et al (2021) Distinct fission signatures predict mitochondrial degradation or biogenesis. Nature 1-5. https://doi.org/10.1038/s41586-021-03510-6

78.Dwane S, Durack E, Kiely PA (2013) Optimising parameters for the differentiation of SH-SY5Y cells to study cell adhesion and cell migration. BMC Res Notes 6:. https://doi.org/10.1186/1756-0500-6-366 
79.Ashley N, Harris D, Poulton J (2005) Detection of mitochondrial DNA depletion in living human cells using PicoGreen staining. Exp Cell Res 303:432-446. https://doi.org/10.1016/j.yexcr.2004.10.013

80.Mangeol P, Prevo B, Peterman EJG (2016) KymographClear and KymographDirect: Two tools for the automated quantitative analysis of molecular and cellular dynamics using kymographs. Mol Biol Cell 27:1948-1957. https://doi.org/10.1091/mbc.E15-06-0404

81.Neumann S, Chassefeyre R, Campbell GE, Encalada SE (2017) KymoAnalyzer: a software tool for the quantitative analysis of intracellular transport in neurons. Traffic 18:71-88.

https://doi.org/10.1111/tra.12456

82.Jakobs MA, Dimitracopoulos A, Franze K (2019) Kymobutler, a deep learning software for automated kymograph analysis. Elife 8:. https://doi.org/10.7554/eLife.42288

\section{Figures}


A

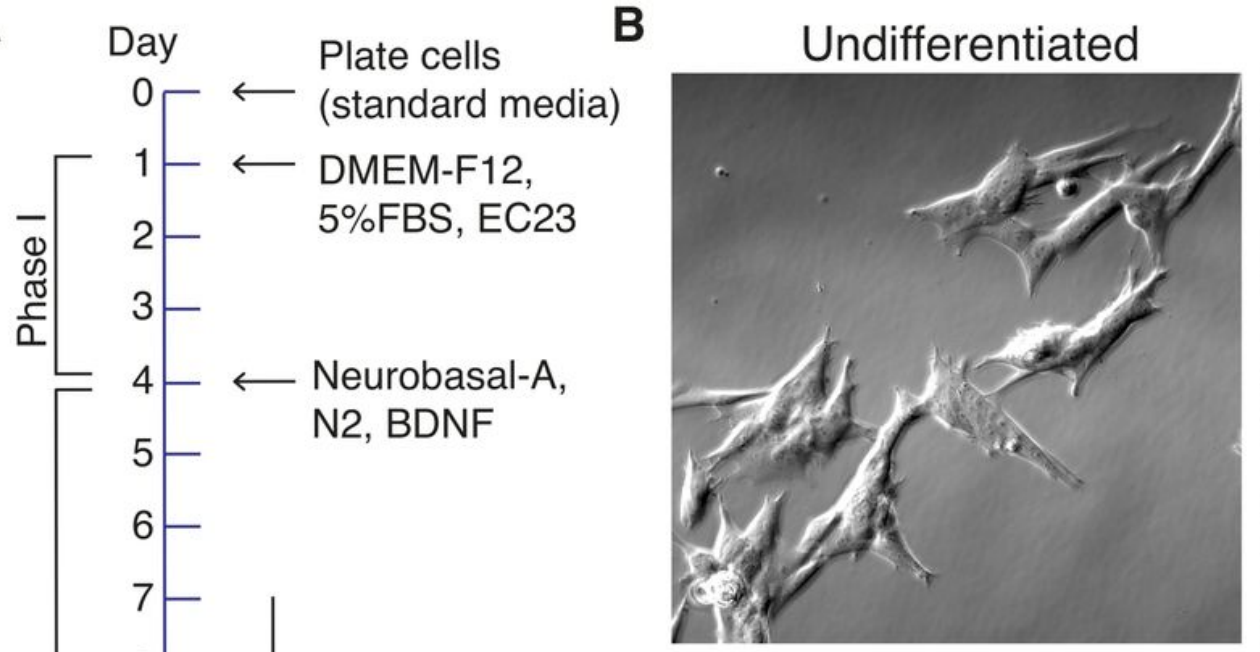

C

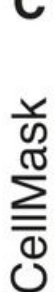

Differentiated phenotype
DIV 14
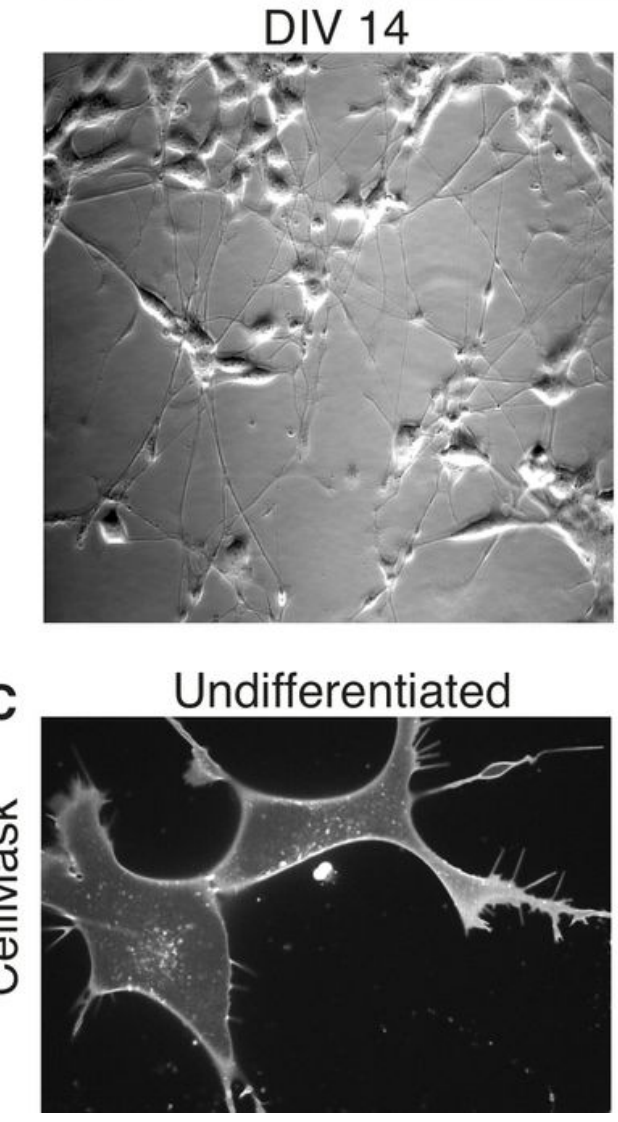

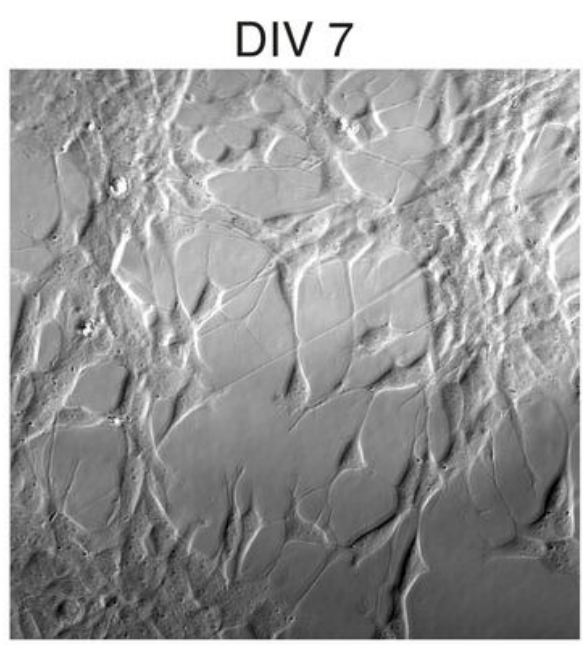

DIV 21

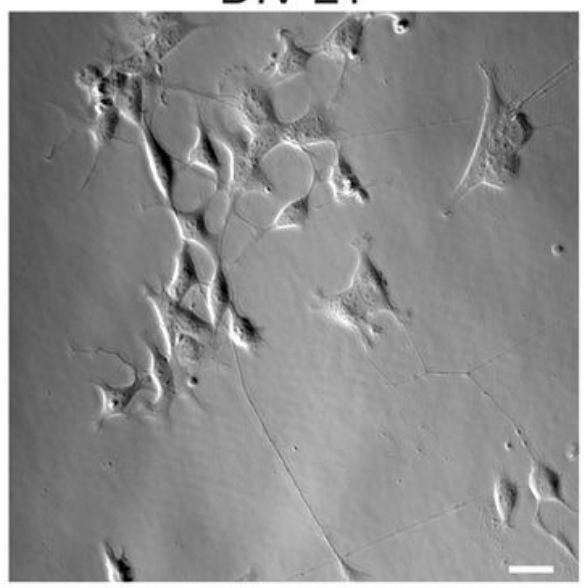

DIV 21

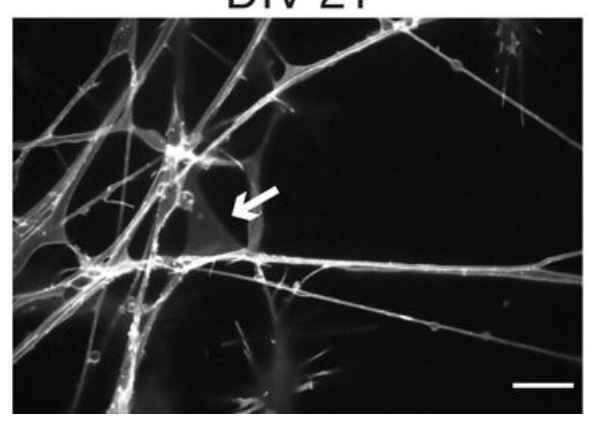

\section{Figure 1}

SH-SY5Y differentiation protocol. (A) Timeline of the SH-SY5Y differentiation protocol outlining the media changes and the different supplements provided. After one week in culture, the cells start displaying a clear neuronal-like phenotype ('Differentiated phenotype'). (B) Representative images of live SH-SY5Y cultures before the start of the differentiation protocol ('Undifferentiated') and during the first (DIV 7), second (DIV 14) and third (DIV 21) week of differentiation. Scale bar: $25 \mu \mathrm{m}$. (C) Higher magnification images of live SH-SY5Y cells stained with the vital dye CellMask Green (CellMask) to mark plasma membrane compartments. Cells at DIV 21 of differentiation display a dramatic change in cellular morphology compared to undifferentiated cells, including the extension of long processes and reduced somal volume. Arrow indicates a cell body. Scale bar: $10 \mu \mathrm{m}$. 

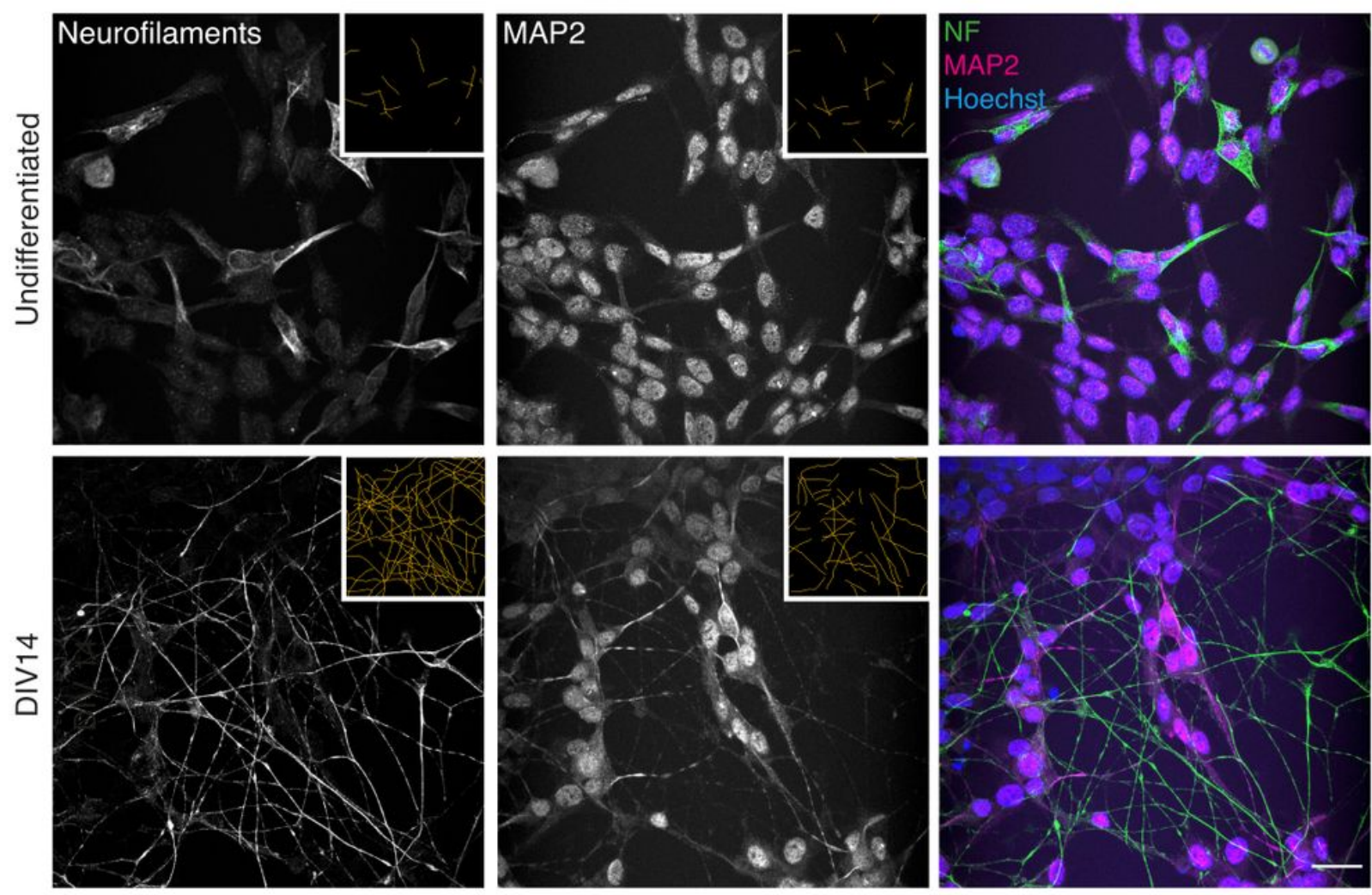

B



C

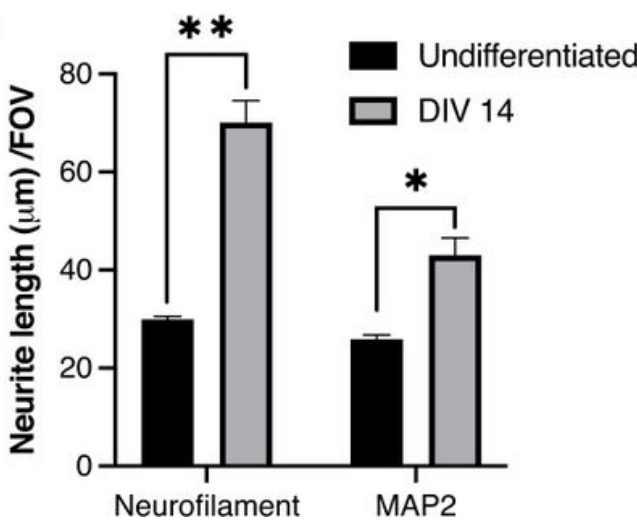

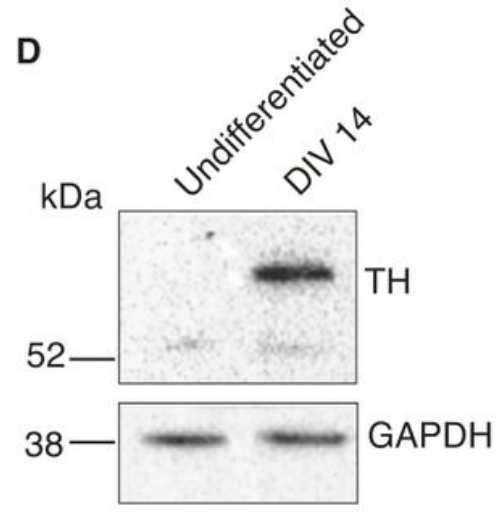

Figure 2

Neuronal markers in differentiated SH-SY5Y cells. (A) Representative images of undifferentiated and DIV 14 cultures stained for neurofilaments and MAP2. Panels on the right show the markers merged with the Hoechst nuclear staining. Inserts show the neurites traced with NeuronJ for each channel. Each image represents a Z-stack projection of 40 and 30 planes for the undifferentiated and DIV 14 images respectively. Scale bars: $25 \mu \mathrm{m}$. (B) Quantification of neurite number and length indicates a higher number of neurites positive for the neurofilament and MAP2 staining in differentiated cells. (C) Neurofilament and MAP2-positive neurites are significantly longer in DIV 14 cells. In (B) and (C), quantifications were done on 8 fields of view (FOV) of $212.13 \mu \mathrm{m}$ by $212.13 \mu \mathrm{m}$. Data are presented as mean \pm s.e.m. ${ }^{*} \mathrm{P} \leq 0.05$; ${ }^{\star *} \mathrm{P} \leq 0.01$, Multiple t-tests. (D) Western blot showing upregulation of tyrosine hydroxylase $(\mathrm{TH})$ in differentiated SH-SY5Ys compared to undifferentiated cultures. GAPDH is used as loading control. 

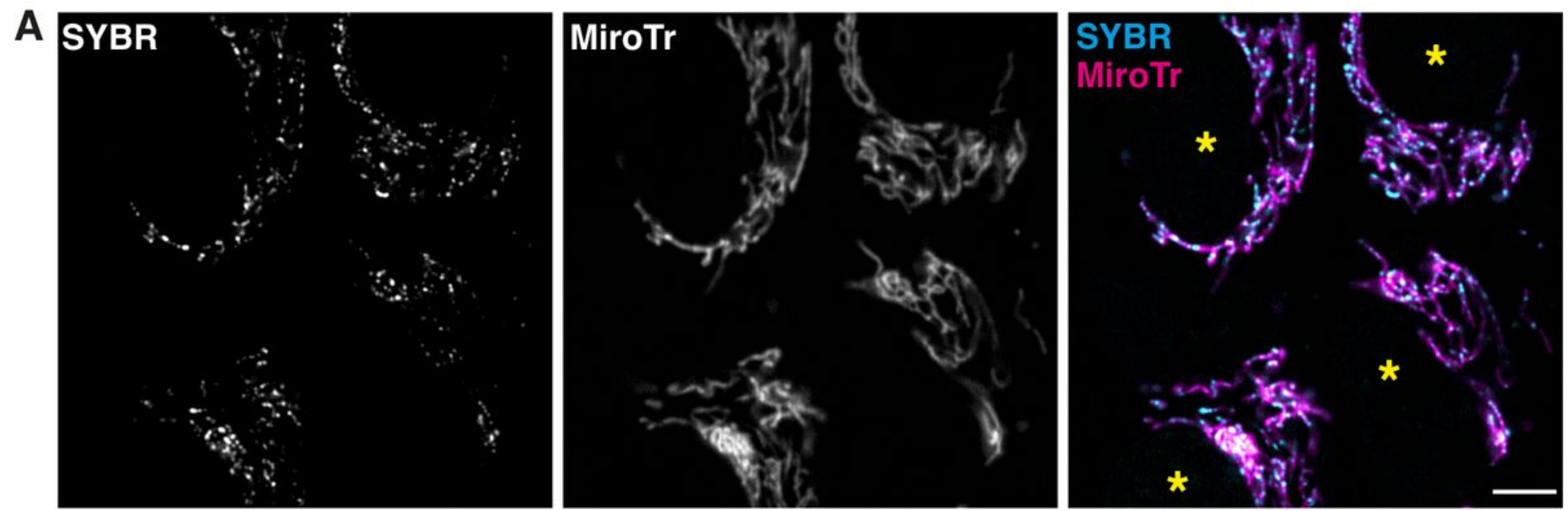

B
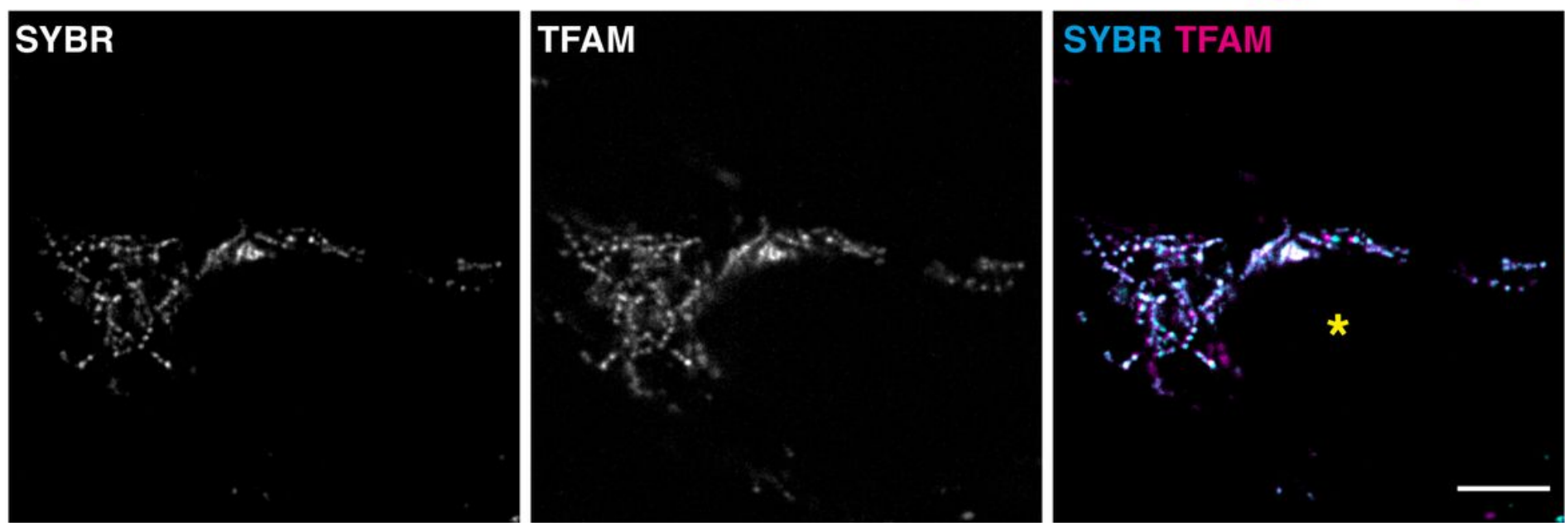

Figure 3

Visualisation of mitochondria and nucleoids in live undifferentiated SH-SY5Y cells. (A) SH-SY5Y cells were stained with SYBR Gold (SYBR) and Mitotracker Deep Red (MitoTr) to visualise the mtDNA and the mitochondria, respectively. The merge pseudo-coloured image shows the distribution of mtDNA puncta (SYBR, cyan) within the mitochondrial network (MitoTr, magenta). (B) Cells were transfected with TFAM m-Scarlet to mark nucleoids and stained with SYBR Gold to visualise the mtDNA. Right panel: merge image showing extensive co-localisation between TFAM (magenta) and mtDNA (SYBR, cyan). Yellow asterisks indicate the position of the nuclei. Scale bars: $5 \mu \mathrm{m}$. 
A
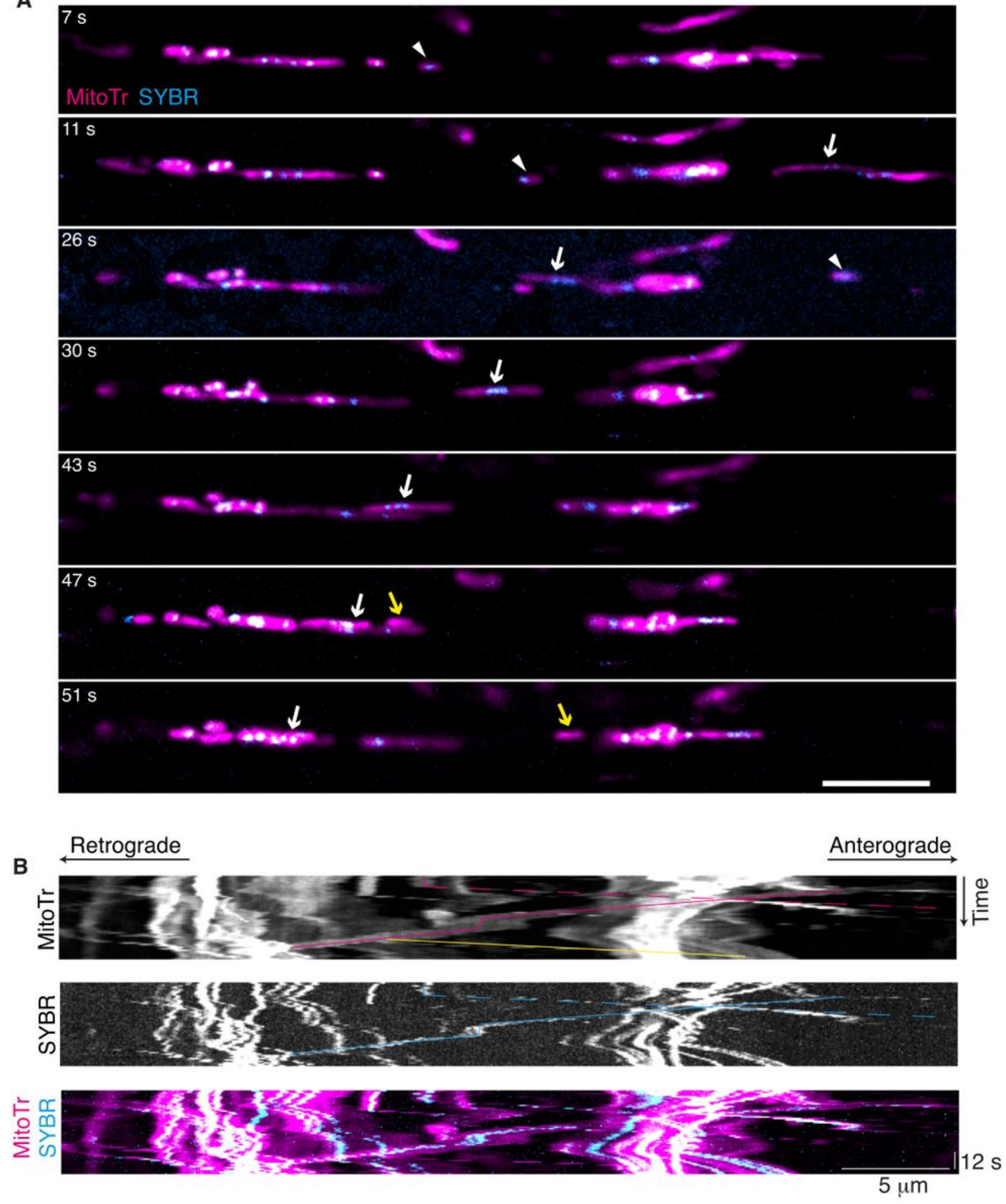

Figure 4

Visualisation of mitochondria and nucleoids dynamics in neurites of differentiated SH-SY5Y (relative to Movie 1). Cells were stained with SYBR Gold (SYBR) and Mitotracker Deep Red (MitoTr) to visualise the mtDNA and the mitochondria, respectively. (A) Still frames showing mitochondria moving in a neurite of DIV 14 cells. The arrowhead indicates a unidirectional movement of a mitochondrion (magenta) containing a nucleoid punctum (cyan). The white arrow shows an example of a mitochondrion that 
undergoes fission during transport (47 s), with the two resulting mitochondria (white and yellow arrows) moving in opposite directions. The nucleoid puncta are partitioned within the same mitochondrion after fission, with one mitochondrion seemingly devoid of mtDNA (yellow arrow). Scale bar: $5 \mu \mathrm{m}$. (B) Kymographs of mitochondrial (MitoTr) and mtDNA (SYBR) dynamics. The dashed lines in magenta (MitoTr) and cyan (SYBR) highlight the mitochondrial and mtDNA movements, respectively, as indicated by the arrowhead in $(A)$, while the continuous lines of the same colours highlight the mitochondrial and mtDNA movements as indicated by the white arrow in (A). The yellow line in the MitoTr kymograph marks the formation and the movement of a mitochondrion generated after a fission event (yellow arrow in (A)). The same track is not present in the SYBR kymograph, suggesting that no mtDNA is partitioned in this mitochondrion. 

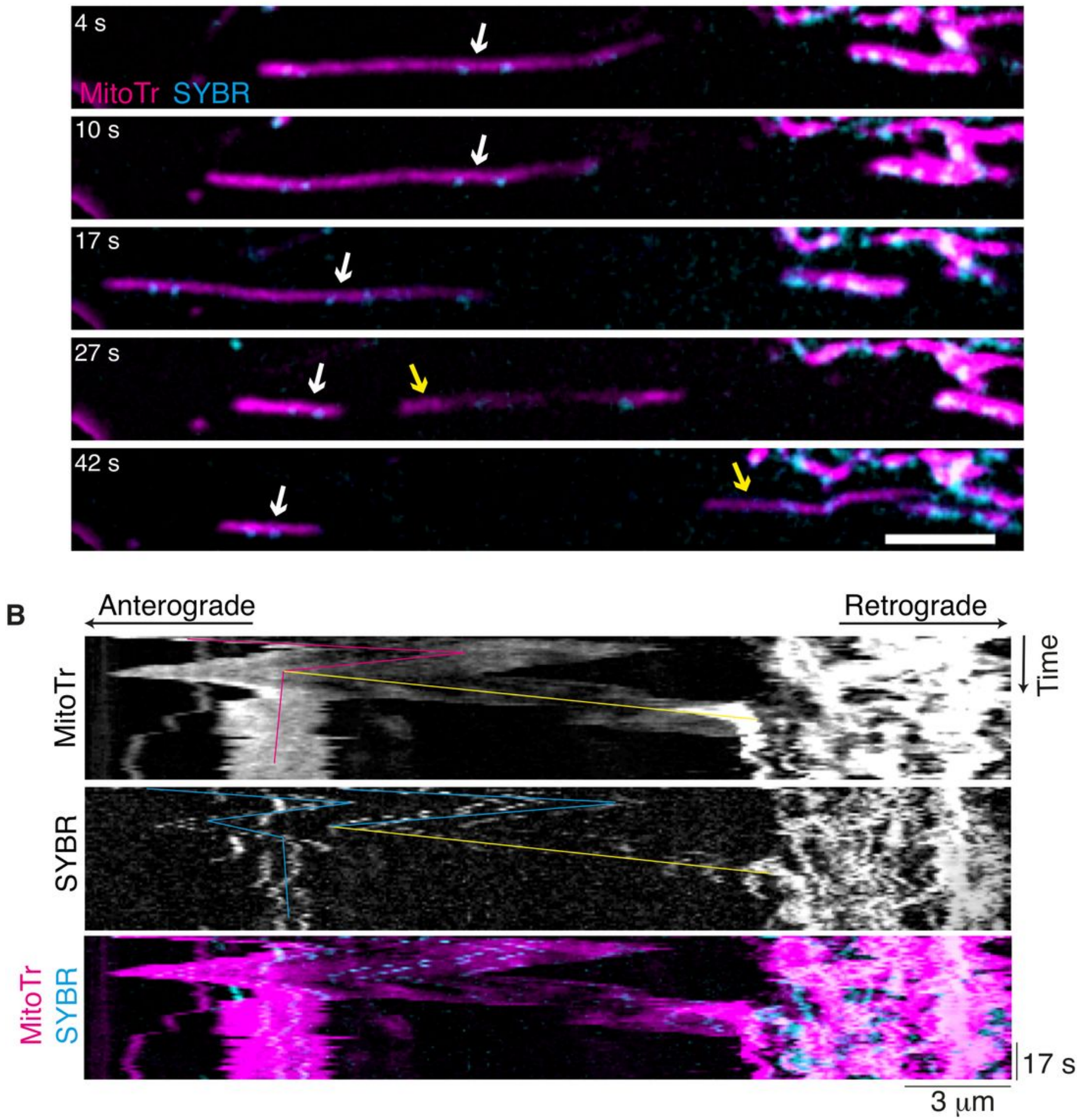

Figure 5

Visualisation of mitochondria and nucleoids dynamics in neurites of differentiated SH-SY5Y cells (relative to Movie 2). Cells were stained with SYBR Gold (SYBR) and Mitotracker Deep Red (MitoTr) to visualise the mtDNA and the mitochondria, respectively. (A) Still frames showing a mitochondrion undergoing fission (27 s) in a neurite of DIV 14 cells, with the two resulting mitochondria (white and yellow arrows) displaying different dynamics. Contrarily to what showed in Figure 4, the nucleoid puncta 
are partitioned within both mitochondria after fission. Scale bar: $3 \mu \mathrm{m}$. (B) Kymographs of mitochondrial (MitoTr) and mtDNA (SYBR) dynamics. The magenta (MitoTr) and cyan (SYBR) lines highlight the coordinated mitochondrial and mtDNA movements, respectively, as indicated by the white arrow in (A). The yellow lines mark the formation and the movement of a mitochondrion (MitoTr) generated after a fission (yellow arrow in (A)), which includes a subset of nucleoids (SYBR) deriving from the original mitochondrion.

\section{Supplementary Files}

This is a list of supplementary files associated with this preprint. Click to download.

- Movie1.mov

- Movie2.mov 